Revue internationale d'éducation de Sèvres

\title{
Europe : désaffection pour les filières scientifiques
}

\section{Cécile de Bouttemont}

\section{OpenEdition}

Journals

Édition électronique

URL : https://journals.openedition.org/ries/1781

DOI : 10.4000/ries. 1781

ISSN : 2261-4265

\section{Éditeur}

France Education international

\section{Édition imprimée}

Date de publication : 1 avril 2002

Pagination : 12-14

ISBN : 2-84520-553-8

ISSN : 1254-4590

\section{Référence électronique}

Cécile de Bouttemont, «Europe : désaffection pour les filières scientifiques », Revue internationale d'éducation de Sèvres [En ligne], 29 | avril 2002, mis en ligne le 01 avril 2005, consulté le 07 juillet 2021. URL : http://journals.openedition.org/ries/1781 ; DOI : https://doi.org/10.4000/ries.1781

Ce document a été généré automatiquement le 7 juillet 2021

(c) Tous droits réservés 


\title{
Europe : désaffection pour les filières scientifiques
}

\author{
Cécile de Bouttemont
}

1 Depuis quelques années, les établissements universitaires scientifiques européens enregistrent une baisse inquiétante de leurs effectifs selon le poids respectif du secteur industriel, et par conséquent des filières correspondantes, dans chacun des pays. Ainsi, en Allemagne et aux Pays-Bas, les effectifs ont été divisés par deux depuis 1996 en physique et mathématique pure ${ }^{1}$. En Belgique, la baisse atteint plus de $30 \%$ sur la même période en ingénierie civile et industrielle. En France, dans les filières universitaires, la baisse est moindre mais reste très significative, avec des disparités fortes entre les matières : chute de $12 \%$ pour la physique ${ }^{2}, 5,8 \%$ en chimie et $2,5 \%$ pour les mathématiques sur les cinq dernières années ${ }^{3}$. En Grande-Bretagne enfin, la rentrée 2000 a connu un recul plus ciblé en microbiologie $(-14 \%)$ et en chimie $(-8 \%)^{4}$.

2 Pour expliquer ce phénomène, la faiblesse de l'enseignement est parfois avancée. D'après Philippe Busquin, commissaire européen pour la Recherche, le problème se situe à trois niveaux: "Le nombre d'heures consacrées aux sciences a sensiblement diminué dans la plupart des pays d'Europe, de même que les moyens financiers, alors que les cours en réclament, ne serait-ce que pour proposer aux élèves un maximum de manipulations. Enfin, faute peut-être de crédits, l'enseignement est trop théorique, alors qu'il faudrait restituer à la science sa dimension intuitive. $\|^{5}$

Cette désaffection peut aussi trouver sa source dans les perspectives de carrières limitées qu'offrent les filières scientifiques aux enseignants-chercheurs. Actuellement, le nombre de postes offerts est relativement réduit. Ainsi, en France, $20 \%$ des jeunes docteurs, toutes filières confondues, ayant soutenu leur thèse dans les dix-huit mois précédents, ont obtenu un poste dans l'enseignement supérieur ou la recherche publique $^{6}$. En Allemagne, le système d'habilitation est un frein à l'engagement de jeunes chercheurs dans la recherche. Par ailleurs, les dépenses consacrées à la recherche sont en constante diminution, ce qui rend les filières scientifiques moins attrayantes. Dans l'Union Européenne, à l'exception des pays nordiques 7 , la part du PIB destinée à la 
science, déjà très inférieure à celle des États-Unis et du Japon, (qui consacrent respectivement $2,7 \%$ et $3 \%$ de leur PIB) a ainsi diminué de $8 \%$ entre 1990 et $1996^{8}$.

Enfin, les filières scientifiques souffrent de la concurrence des filières informatiques qui connaissent un certain engouement avec le développement d'Internet ( $+50 \%$ en Grande-Bretagne cette année, $+22 \%$ en France en 1999$)^{9}$ et des filières commerciales supposées offrir des perspectives de carrière plus attrayantes.

5 Cette désaffection, conjuguée au départ massif à la retraite d'enseignants et au départ de scientifiques pour les États-Unis où ils espèrent des carrières plus prometteuses, risque d'entraîner à moyen terme une pénurie d'enseignants-chercheurs dans les disciplines scientifiques et à plus long terme de pénaliser les capacités de l'Union Européenne à mener des recherches de pointe. Pour juguler la baisse importante des effectifs, plusieurs gouvernements européens envisagent de prendre diverses initiatives, qui s'articulent autour des cinq axes suivants.

\section{Revaloriser les carrières scientifiques et réduire la fuite des cerveaux}

6 Ainsi aux Pays-Bas, les salaires dans le secondaire et l'université ont été augmentés. En Grande-Bretagne, le gouvernement souhaite mettre en place des récompenses financières et des systèmes de bourses afin de résoudre le problème de recrutement et de maintien en poste des équipes universitaires ${ }^{10}$. En Allemagne, trois centres de recherches vont être créés au sein des universités avec pour objectif d'accroître l'attractivité de la recherche universitaire allemande. Des mesures gouvernementales d'un montant de 85 millions d'euros sont destinées à internationaliser le paysage scientifique allemand dans le cadre du « programme d'investissement pour le futur ».

\section{Simplifier l'accès à la recherche}

7 En Allemagne, en particulier, une loi sur le déroulement des carrières à l'université, devrait accorder moins d'importance à l'habilitation. Ce nouveau règlement devrait permettre de raccourcir le délai entre le doctorat, la période post-doctorale, la dernière phase de l'habilitation et l'obtention éventuelle de moyens pour diriger une équipe de recherche.

\section{Mener une politique volontariste de promotion des sciences}

8 Et contrebalancer l'ère économiste actuelle. Alors que l'Europe dépasse les États-Unis en terme de publications scientifiques, la diffusion des travaux de recherche reste cantonnée au niveau national ${ }^{11}$. Les organismes de recherche sont fortement incités à renforcer la communication sur leurs travaux. Par ailleurs, pour sensibiliser la population aux sciences, le gouvernement néerlandais a créé la fondation Axis, chargée de coordonner et d'organiser des campagnes de promotion des sciences. Du même ordre, le projet « La Main à la pâte » en France, s'adresse aux enfants du primaire. 


\section{Améliorer l'enseignement des sciences à l'école}

Une refonte des études de sciences de la maternelle à l'université est ainsi envisagée en France. Par ailleurs, l'enseignement devrait être systématiquement intégré dans les carrières des chercheurs.

\section{Au niveau européen, accroître la mobilité des chercheurs et ouvrir les universités à l'international}

10 L'Union européenne envisage de doubler le nombre des chercheurs bénéficiant de bourses européennes. Les effets d'une telle mesure sont apparus dès 1999 : le nombre de post-doctorants présents dans les pays de l'Union européenne a dépassé le nombre de ceux qui ont choisi les États-Unis ${ }^{12}$, des projets permettant aux étudiants en thèse d'être accueillis dans les laboratoires européens pour des durées de plusieurs mois pourraient voir le jour.

\section{NOTES}

1. «Bulletin scientifique des ambassades », AEF, 03/01/01.

2. RDTInfo, novembre 2001, nº́spécial, p. 8

3. «Filières scientifiques recherchent étudiants ", Le Progrès, 16/10/01, p. 8

4. AEF, 26/10/01.

5. RDTInfo, novembre 2001, n spécial, p. 3.

6. La Lettre de l'OST, hiver 99-2000, $\mathrm{n}^{\circ} 18, \mathrm{p} .5$.

7. La Suède, en tête des pays de l'Union, consacre 3,6 \% de son PIB à la RetD.

8. La part de la France est de $2,3 \%$ et reste au-dessus de la moyenne européenne de 1,9\%. Sources : EUROSTAT.

9. «Filières scientifiques recherchent étudiants », Le Progrès, 16/10/01, p. 8.

10. Le gouvernement britannique vient de débloquer 4 millions de $€$ (43 MF) en janvier 2001 . Source : AEF, 03/01/01.

11. Id.

12. La Lettre d'Egide, janvier 2001, n²1, p. 5.

\section{INDEX}

Index géographique : Europe 
AUTEUR

CÉCILE DE BOUTTEMONT

Documentaliste au Centre de ressources documentaires du CIEP 